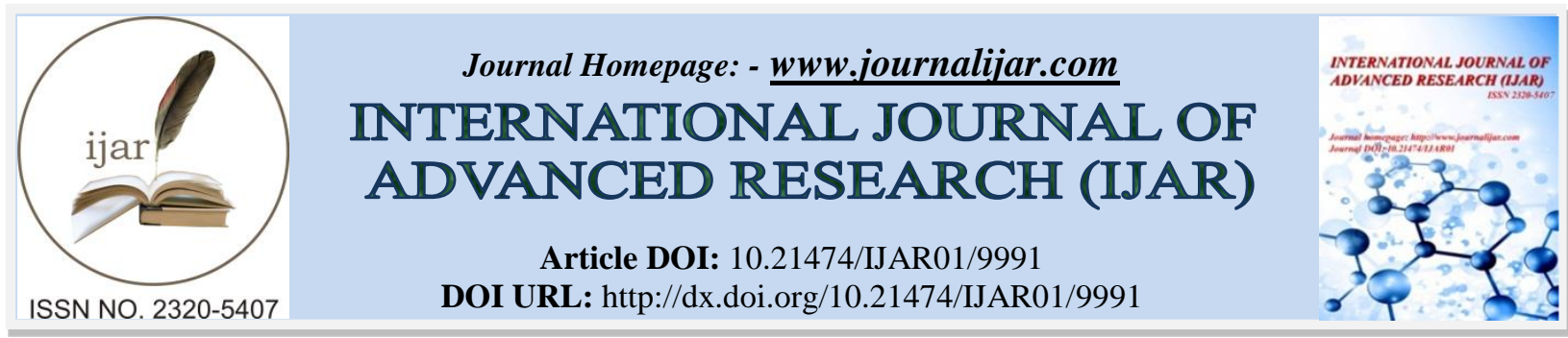

RESEARCH ARTICLE

\title{
HYPERTENSION PORTALE ET GROSSESSE : EXPERIENCE DU SERVICE DE GYNECOLOGIE- OBSTETRIQUE II DU CHU HASSAN II DE FES, A PROPOS DE CINQ CAS COLLIGES DURANT L'ANNEE 2017-2018.
}

Nyingone S, Kriouile M, Chaara H, Fdili Alaoui F-Z, Jayi S and Melhouf. Centre Hospitalier et Universitaire Hassan II de Fès ; Faculté de Médecine et Pharmacie de Fès.B.P : 1893, KM 2.200, Rte Sidi Harazem, Fès 30070, Maroc.

\section{Manuscript Info}

\section{Manuscript History}

Received: 05 September 2019

Final Accepted: 07 October 2019

Published: November 2019

Key words:-

Portal hypertension- pregnancymanagement.

\begin{abstract}
Portal hypertension on cirrhotic liver or not, when it is well supported; improves the vital prognosis in view of the hemorrhagic complications that it incurs. Thus, any woman before conception should consult her gastroenterologist in order to predispose to an armed follow-up in association with an obstetrician. Because the presence of a pregnancy in a woman with portal hypertension is a high-risk period, which can have a maternal-fetal prognosis. This risk can lead to maternal death, miscarriage, fetal death in utero or even prematurity or peri-and postnatal death. However, primary prevention is proposed in these patients, in order to act upstream on esophageal varices either by cardi-selective beta-blockers or by endoscopic ligation. The modalities of delivery will be discussed as appropriate. Although the lower lane is preferred, caesarean section also has its place, especially in purely obstetric indications. Thus the coalition of anesthesia staff, of neonatology in concomitance with gastroenterologists and obstetricians would be codified. For it will also be necessary to discuss the type of analgesia for which delivery route.

To this end, we wanted to highlight our experience on the peculiarity of management of these pregnancy states collected within the gynecologyobstetrics department II of CHU Hassan II of Fes about five patients. A prospective study followed during the year 2017-2018. Two of the women were treated in delivery room with a strict minimum balance, while the other three were well followed with multidisciplinary care in collaboration with gastroenterologists, anesthetists and pediatricians.
\end{abstract}

Copy Right, IJAR, 2019,. All rights reserved.

\section{Introduction:-}

L'hypertension portale(HTP) est définie comme une augmentation de la différence de pression entre le système porte et le système cave qui est de $5 \mathrm{mmHg}$. Elle explique mieux le développement de la circulation collatérale porto-systémique et l'hémorragie digestive. Les manifestations cliniques apparaissent lorsque la différence de pression est au-delà de $10 \mathrm{mmHg}$. La grossesse vient également accroitre ce risque par la majoration physiologique de la pression portale observée à partir du deuxième trimestre où l'expansion volémique est à son maximum, et pouvant être responsable d'une hémorragie digestive par rupture des varices œsophagiennes. C'est la complication la plus redoutable qui met en jeu le pronostic materno-fœtal. D'où le terme de grossesse à risque, car l'association

Corresponding Author:- Nyingone S.

Address:- Centre Hospitalier et Universitaire Hassan II de Fès ; Faculté de Médecine et Pharmacie de Fès. 177 B.P : 1893, KM 2.200, Rte Sidi Harazem, Fès 30070, Maroc. 
hypertension portale et grossesse peut entrainer de fausses couches, des morts fœtales in utero voire une prématurité. De ce fait, sa prise en charge devient alors multidisciplinaire mettant en action obstétriciens, hépato-gastroentérologues, pédiatres néonatologues et anesthésistes. Ainsi chez les femmes porteuses d'une hypertension portale, la grossesse devrait être planifiée, et l'hypertension portale connue (cirrhotique ou non), prise en charge avant toute conception. La prévention primaire sur les varices œsophagiennes semble améliorer le pronostic fœeto-maternel ; d'où l'intérêt de réaliser une endoscopie digestive pour dépister les types de varices œsophagiennes et d'agir en amont soit par des bêta-bloquants non cardio-sélectifs ou par ligature.

Les modalités d'accouchement seront ainsi discutées en fonction de la gravité de la pathologie. L'accouchement voie basse semble le meilleur abord et la césarienne avec ses risques infectieux et hémorragiques n'est pas recommandée de manière systématique; elle ne devrait être réservée qu'aux indications purement obstétricales.

C'est ainsi que nous nous sommes penchés sur la particularité de prise en charge de ces états gravidiques à risque au sein de notre formation de gynécologie obstétrique II au CHU Hassan II de Fès : à propos de 5 cas. Notons que trois patientes nous ont été adressées avec le maximum de bilans réalisés, ce qui a permis un meilleur suivi en consultation prénatale et les deux autres sont des parturientes, prises directement en charge à la salle d'accouchement.

\section{Observation et Patientes :-}

Observation $\mathrm{n}^{\circ} 1$ : Mme A.A, âgée de 28ans, de bas niveau socio-économique, sans habitude toxique, primipare, référée pour prise en charge d'une grossesse initialement à 10SA(Semaines d'aménorrhée) chez une femme porteuse d'une HTP $(175 \mathrm{~mm})$ d'étiologie indéterminée sur foie cirrhotique sans lésion focale, en décompensation ascitique et hémorragique ayant bénéficié d'une ligature de varices (stade II avant conception) et diurétiques pour assèchement d'ascite puis mise sous propranolol en prévention secondaire. L'examen trouve des conjonctives décolorées, une énorme splénomégalie $(22 \mathrm{~cm})$, avec Syndrome d'épanchement, sans syndrome hémorragique ni ictère. La FOGD montre une varice œsophagienne stade I sans signe rouge, au bilan biologique : les sérologies hépatitiques $\mathrm{C}$ et $\mathrm{B}$ négatives, bilan d'auto-immunité négatif, avec une bicytopénie constante au cours de la surveillance ( $\mathrm{Hb}$ à 6.8 hypochrome microcytaire ferriprive; Plaquettes à 58000 éléments sur frottis), Taux de prothrombine à $75 \%$; sans perturbation du bilan hépatique ni retentissement sur le fotus. Patiente suivie concomitamment avec les gastro-hépato-entérologues, l'accouchement voie basse a été accepté. La grossesse a évolué jusqu'à 39SA où une échographie obstétricale a mis en évidence un oligoamnios sévère, vessie et reins sans anomalie sur une grossesse monofoetale évolutive, et un placenta grade III de Granum calcifié sans notion d'hydrorrhée. Une césarienne a été indiquée en concertation avec les hépato-gastro-entérologues, les réanimateurs anesthésistes et les néonatologues; faite sous rachi-anesthésie, avec ascite de moyenne abondance $1000 \mathrm{ml}$ et une énorme splénomégalie atteignant l'ombilic. Transfusée en per-opératoire par 6 culots plaquettaires et 3 culots globulaires A+ phénotypés avec $\mathrm{Hb}$ de contrôle à 9.5, plaquettes à 83000. Les suites post-partum se sont déroulées sans complications.

Observation $n^{\circ} 2$ : Mme R.D, âgée de 29ans, de niveau socio-économique bas, sans habitude toxique, ni prise médicamenteuse, $2^{\text {ème }}$ geste, avec une fausse couche spontanée à 2 mois de conception au cours d'une hémorragie digestive sur varices stade II, référée pour prise en charge d'une grossesse de 37SA chez une femme connue porteuse d'une HTP $(190 \mathrm{~mm})$ de cause inconnue sur foie cirrhotique sans lésion suspecte (sérologies hépatitiques B et $\mathrm{C}$ négatives) avec varices œsophagiennes stade II mise sous propranolol et découverte au $2^{\text {ème }}$ trimestre d'un diabète gestationnel déséquilibré à $60 \%$ sous insulinothérapie. L'examen trouve une énorme splénomégalie de $23 \mathrm{~cm}$ avec circulation veineuse collatérale, et syndrome ascitique et une hauteur utérine correspondant au terme. Bilans biologiques : thrombopénie à 55000 éléments, $\mathrm{Hb}$ à $10.1 \mathrm{~g} / \mathrm{dl}$, Taux de prothrombine à $90 \%$, le reste du bilan hépatique et de retentissement sans anomalie. A l'échographie obstétricale : une grossesse monofoetale évolutive en présentation céphalique et une tendance à la macrosomie avec une estimation du poids foetal à $3540 \mathrm{~g}$, le doppler ombilical et utérin normal. Elle a été hospitalisée pour modalité d'accouchement. Un avis anesthésiste et gastroentérologue préconisait une voie basse si taux de plaquettes $>50000$. Durant la surveillance, jusqu'à 38SA, Cycle glycémique toujours perturbé avec NFS en faveur d'une thrombopénie à 45000 sur frottis. Une voie haute a été indiquée sous Anesthésie générale, découverte d'une ascite de moyenne abondance $(800 \mathrm{ml})$ et une énorme splénomégalie arrivant jusqu'à l'ombilic, extraction d'un nouveau-né de sexe masculin, poids à la naissance $3600 \mathrm{~g}$. Transfusée en per-acte par 8 culots plaquettaires et 1 culot globulaire A+ phénotypé. Les suites post-césariennes étaient sans anomalies. 
Observation $\mathrm{n}^{\circ} 3$ : Mme F.N, âgée de 33 ans, de niveau socio-économique faible, $3^{\text {ème }}$ geste, $3^{\text {ème }}$ pare tous des accouchements par voie basse dont deux compliqués d'une hémorragie de la délivrance jugulée médicalement, adressée dans notre formation pour prise en charge d'une HTP sur cavernome porte avec foie non cirrhotique et une splénomégalie $(17 \mathrm{~cm})$ sur une grossesse initialement de $12 \mathrm{SA}$ contractée sous pilule oestroprogestative, sans aucun traitement médical. Grossesse bien suivie en obstétrique en collaboration avec les gastro-entérologues et les réanimateurs jusqu'à 39SA, sans retentissement fotal ni décompensation ascitique ou hémorragique; avec à l'examen une splénomégalie. La FOGD objectivait des varices œsophagiennes stade I sans signes rouges. Au bilan biologique : une thrombopénie évolutive 64000/58000/47000; TP à 82\%; avec bilan de thrombophilie perturbé (antithrombine, homocystéine, protéine $\mathrm{S}$, et $\mathrm{C}$ diminuées), sérologies hépatitiques $\mathrm{B}$ et $\mathrm{C}$ négatives, le reste du bilan prénatal et de fin grossesse sans anomalie. Un accouchement par voie basse sous péridurale aidé par une extraction instrumentale (ventouse) et une épisiotomie, sans complications per ou post-partum. Transfusée au préalable par 8 culots plaquettaires.

Observation $n^{\circ} 4$ et 5 :

Patientes $\mathrm{n}^{\circ} 4$ et 5 sont des grandes multipares, de bas niveaux socio-économiques, sans habitudes toxiques, enceintes à terme, des grossesses non suivies, et dont la découverte d'une hypertension portale sur foie non cirrhotique était fortuite sur une échographie abdominale demandée par le médecin référent devant une splénomégalie. Arrivée aux urgences en début de phase active du travail, avec à l'examen aucun signe de décompensation ascitique ou hémorragique, palpation d'une splénomégalie. Bruits cardiaques fœtaux bien perçus et réguliers et un rythme cardiaque fotal oscillant et réactif, échographie obstétricale sans anomalie décelée. Bilans biologiques : thrombopénie à 62000 et 71000 éléments; sérologies hépatites $\mathrm{B}$ et $\mathrm{C}$ négatives, bilan d'auto-immunité non fait par manque de moyens. Accouchements voie basse sous péridurale avec ventouse, donnant naissance à des nouveau-nés bien portants ; les suites de couches immédiates et ultérieures sans incident. Transfusées en per-partum par 8 et 6 culots plaquettaires. Patientes adressées en gastro-entérologie pour complément de prise en charge. Mais perdues de vue jusqu'à ce jour.

\section{Discussion :-}

L'hypertension portale définie comme une augmentation des pressions du réseau veineux porte, qui dépassant un seuil, peut entrainer des complications comme la survenue d'une ascite, d'une hémorragie digestive, un hypersplénisme mettant en jeu le pronostic vital.

La présence d'une grossesse chez une femme porteuse d'une hypertension portale est une situation à risque aussi bien sur le plan foetal que maternel. Compte tenu des complications engendrées par l'hypertension portale telles, les hémorragies digestives qui surviennent par rupture de varices œesophagiennes et engagent ainsi le pronostic maternel qui peut être un décès maternel ; et le pronostic fœtal allant d'une fausse couche, un avortement tardif, une mort fœtale in utéro, une prématurité à un décès péri ou post-natal $\left[\mathbf{1 , 2 , 3 , 4 ]}\right.$. Notre patiente $\mathrm{n}^{\circ} 2$ dans ses antécédents évoque la notion de fausse couche concomitante à la survenue d'une hémorragie digestive par rupture de varices œsophagiennes stade II, avant la conception actuelle. Par ailleurs, ce symptôme redoutable n'a pas été rapporté dans notre étude comme dans celles de plusieurs auteurs $\left[\begin{array}{l}1,2,5,6,7,8 \\ \mathbf{2}\end{array} \mathbf{6}\right.$ mais il est à craindre dans la surveillance de l'évolution de la grossesse.

L'hypertension portale est une pathologie qui concerne pays industrialisés que pays en voie de développement. Elle peut survenir sur une cirrhose de foie ou sur un foie non cirrhotique et dont les causes seront connues ou indéterminées. En effet la démarche diagnostique devrait s'articuler à ressortir dans l'anamnèse, une notion de prise médicamenteuse, de toxiques, une consommation d'alcool ; la cirrhose étant la première cause de l'hypertension portale [9-13]. Nous ressortons trois(3) cas de foie non cirrhotique : parmi eux, un sur thrombose porte (cavernome chez patiente $\mathrm{n}^{\circ} 3$ ) et deux(2) cas de cirrhose du foie d'origine indéterminée (cas $\mathrm{n}^{\circ} 1$ et 2 ). Nos patientes avaient toutes bénéficié de sérologies hépatitiques revenues toutes négatives, éliminant une implication virale $\mathrm{B}$ et $\mathrm{C}$ qui sont fréquentes dans la cause des foies cirrhotiques en dehors de l'alcoolisme, un facteur non retrouvé dans l'anamnèse de nos patientes. Ce qui devrait attirer une attention particulière sur des foies non cirrhotiques en pays Maghrébins, car beaucoup d'études faites dans ces pays soulèvent ainsi la survenue d'une cirrhose de foie sur terrain d'hépatite C ou B [14-18]. Par contre l'ascite et l'hypersplénisme prennent bien leur place dans les complications de l'HTP ; toutes nos patientes présentaient une thrombopénie dont une avec une bicytopénie (cas $\mathrm{n}^{\circ} 1$ ), et l'examen trouvait une splénomégalie bien qu'elle ne soit pas toujours un signe direct d'hypersplénisme. L'ascite est présente chez deux de nos patientes porteuses d'une cirrhose de foie, complication très fréquente dans l'hypertension portale 
sur foie cirrhotique du point de vue épidémiologique et qui s'exprime chez $60 \%$ des patients atteints de cirrhose [19]. Cependant elle est rare en cas de grossesse du fait de l'augmentation de la pression abdominale; ce que confirme l'examen de trois de nos patientes $\left(\mathrm{n}^{\circ} 3,4\right.$ et 5 ). Ainsi pour une meilleure prise en charge, l'hypertension portale chez une femme enceinte nécessite une étroite surveillance et des investigations adéquates vue sa délicatesse, faisant intervenir différents protagonistes médicaux à savoir obstétriciens, gastro-hépato-entérologues, réanimateur et pédiatres néonatologues. La prophylaxie des complications hémorragiques de l'hypertension portale est basée soit sur la chirurgie des varices, soit sur des bétabloquants cardio-sélectifs. Une de nos patientes avait bénéficié d'une ligature de varices esophagiennes stade II avant sa conception devant la survenue d'une hémorragie digestive. Tandis que dans l'ensemble seules deux sont sous bétabloquants (Propranolol), indiqués par les gastro-entérologues pour une éventuelle prévention des hémorragies digestives par rupture de varices. Remarquons que trois de nos patientes bien suivies ont bénéficié malgré leur niveau socio-économique, d'un minimum d'investigations telles une fibroscopie stadifiant les varices et permettant une décision thérapeutique ou non. Par ailleurs, il est annoncé de la littérature que les ruptures de varices œsophagiennes survenaient sur 38\% de patientes cirrhotiques enceintes; et que les cirrhotiques porteuses de varices œsophagiennes présenteraient dans $78 \%$, un risque de rupture de leurs varices au cours de la grossesse [20]. Notre étude ne rapporte aucune rupture de varices œsophagiennes que ce soit chez les femmes cirrhotiques que les non cirrhotiques pendant ou avant l'accouchement. De même il a été établi que les patientes ayant bénéficié avant leur grossesse d'un traitement de leurs varices ont eu moins de complications pendant la délivrance et un faible taux de mortalité fotale [21.22]. Toutes nos patientes y compris celle qui a été traitée de ses varices avant la grossesse, ont accouché sans complications. En effet, Maurey et al dans leur étude montrent que, le pronostic materno-foetal est en général meilleur lorsque la patiente a un bon suivi et consulte assez tôt [3]. Et nous pouvons l'attester dans l'ensemble de notre étude, malgré le fait que deux de nos patientes aient été prises en charge en phase active du travail. Parallèlement, le traitement de l'ascite reste à discuter, nous rapportons la présence d'ascite tout au long du suivi de deux de nos cas $\left(\mathrm{n}^{\circ} 1\right.$ et 2$)$. Cependant les diurétiques sont recommandés pour assécher l'ascite lorsqu'elle devient cliniquement significative et doivent être débutés sous observation clinique et biologique [19]. Une seule de nos patientes s'est vu prescrire des diurétiques devant l'aggravation de son ascite en début de grossesse, soldé par un tarissement partiel (cas n ${ }^{\circ} 1$ ). Ceci peut-être expliqué par l'arrêt du traitement après l'amélioration clinique.

La question qui se pose en ce moment est centrée sur les modalités d'accouchement, impliquant une décision d'obstétriciens, gastro-hépatologues et anesthésistes. D'où la nécessité d'une surveillance rapprochée et bien codifiée. Longtemps la césarienne a été la voie d'accouchement de prédilection devant cette complexité hypertension portale et grossesse du fait de la non-maitrise de ces cas [23]. Aujourd'hui il est observé une évolution concernant la prise en charge de ces états gravidiques, qui permet de réserver l'indication de la voie haute pour les causes strictement obstétricales comme le préconisent la plus part des auteurs $[\mathbf{1} ; 3: 7 ; 8,24: 25: 26 ;$ et 27$]$. Notre série mentionne deux cas de césariennes indiquées pour oligoamnios sévère $\left(\operatorname{cas}^{\circ}{ }^{\circ} 1\right)$ et diabète déséquilibré associé à une thrombopénie sévère (cas $\left.\mathrm{n}^{\circ} 2\right)$ en collaboration avec les réanimateurs, les gastro-entérologues et les néonatologues sur des grossesses bien menées à terme. Extractions des nouveau-nés faite, sans incident avec un suivi per et post-partum sans complication. Cette attitude a été murie pour le cas de l'oligoamnios, car n'ayant pas encore à disposition pour les pays africains, des prostaglandines naturelles pour déclencher un travail. La rachianesthésie et l'anesthésie générale ont été indiquées au cas par cas en fonction de l'avis des réanimateurs. Une seule a bénéficié d'une analgésie générale devant une thrombopénie inférieure à 50.000 éléments (45000 pour la patiente $\mathrm{n}^{\circ} 2$ ). La péridurale quant à elle a été pratiquée chez toutes les parturientes ayant accouché par voie basse (cas n 3,4 et 5), avec extractions instrumentalisées par ventouse, de nouveau-nés bien portants. A cet effet, concernant l'accouchement proprement dit, il est recommandé une analgésie maternelle en début de travail, par anesthésie

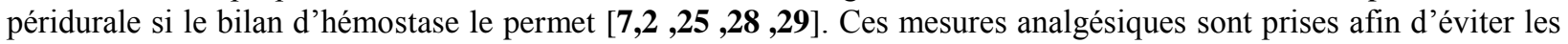
efforts de poussée qui pourront être responsables d'une rupture de varices, ou un éclatement des vaisseaux collatéraux hépato-porte dans le cas du cavernome portal et qui se manifestera par une hémorragie foudroyante, qui peut être fatale pour la parturiente. C'est pour cela une échographie abdominale minima doit être faite devant une symptomatologie digestive (splénomégalie, une ascite, un ictère...) afin de rechercher une origine hépatique, car les cavernomes peuvent être découverts de manière fortuite. La thrombose du tronc porte, peut s'associer à une thrombophilie comme le cas de notre patiente $n^{\circ} 3$. C'est la raison pour laquelle nous insisterons sur l'étroite collaboration entre les spécialistes concernés, afin d'assurer un bon suivi pour toute patiente porteuse d'une hypertension portale, car toutes les décisions convergeront pour le bien-être de la patiente et son foetus. C'est ainsi qu'une césarienne ne sera pas indiquée à tort. Toutefois aucune étude n'a démontré qu'il existait des données précises quant aux complications de l'accouchement par voie basse comparée à la voie haute [3 ,22]. En outre, il est observé que les hémorragies de la délivrance sont plus fréquentes dans l'hypertension portale (30]; et se voient plus 
chez les femmes malades d'une cirrhose dans 8 à $26 \%$ de cas $[\mathbf{1 , 2 , 6 , 7 , 8 ]}$ et favorisées par les troubles de l'hémostase[8]; tandis qu'elles sont rares dans les hypertensions portales non cirrhotiques $(0-4 \%)$ [1,26]. Il est également à éviter la survenue des infections en post-partum car elles semblent fréquentes chez les patientes cirrhotiques [2]. Ainsi le per et post-partum de toutes nos parturientes n'a révélé aucune particularité notamment absence d'hémorragies de la délivrance et d'infection. Il faut aussi signaler qu'aucune d'entre elles n'avaient un trouble de l'hémostase. Par conséquent, pourrons nous aussi expliquer la prévention de ces complications hémorragiques par la transfusion plaquettaire effectuée au préalable chez toutes nos patientes. Bien que certaines études ne conseillent pas la transfusion systématique sauf en cas de thrombopénie sévère inférieure à 50.000 plaquettes pour la césarienne, et moins de 30.000 plaquettes pour la voie basse [31]. Ceci dit nos réalités ne restent pas les mêmes, nous choisissons de prévenir la survenue d'hémorragie du post-partum vu le risque accouru de décès maternels dans les pays en voie de développement.

Actuellement, trois d'entre nos patientes bénéficient d'une surveillance constante en unité de gastro-hépatoentérologie tandis que deux sont malheureusement perdues de vue. Le suivi pré-conceptionnel est important car il améliore le pronostic de ces patientes; et permettra d'agir en amont d'une complication avant la grossesse vu les risques qu'expose l'hypertension portale.

\section{Conclusion :-}

Les complications de l'hypertension portale sont à redouter car l'existence d'une cirrhose ou non prédispose à des risques hémorragiques pouvant interférer dans l'évolution d'une grossesse.

A cet effet, un suivi obstétrical doit impérativement être associé à une surveillance hépatologique stricte et renforcée. Et la césarienne sera murie par les indications purement obstétricales. Par conséquent un bilan étiologique de toute hypertension portale, devrait-être préconisé avant la conception.

\section{Références :-}

1. Cheng YS. Pregnancy and liver cirrhosis and/or portal hypertension. Am J Obstet and Gynecol 1977; 128: 81222.

2. Restaino A, Compabasso C, D’Aloya A, Abbruzzese AD, Valerio A, Pansini F. Cirrhosis and pregnancy. A case report and review of the literature. Clin Exp and Obstet Gynecol 1996; 23:240-7.

3. Maurey A, Bernuau J, Ganne N, Uzzan M, Mandelbrot L, Colau JC, et al. La grossesse est possible chez les femmes atteintes de cirrhose. Gastroenterol Clin Biol 2006 ; 30 : 1064.

4. Rautou PE, Angermayr B, Raffa S, Peck-Radosavljevic M, Garcia-Pagan JC, Moucari R, et al. Maternal and fetal outcome in 27 women with Budd-Chiari syndrome and 41 pregnancies. Hepatology 2007; 46: 736A.

5. Varma RR, Michelsohn NH, Borkowf HI, Lewis JD. Pregnancy in cirrhotic and noncirrhotic portal hypertension. Obstet Gynecol 1977; 50: 217-22.

6. Schreyer P, Caspi E, El-Hindi JM, Eshchar J. Cirrhosis-pregnancy and delivery: a review. Obstet Gynecol Surv 1982; 37: 304-12.

7. Pajor A, Lehoczky D. Pregancy in liver cirrhosis. Assessment of maternal and fetal risks in eleven patients and review of the management. Gynecol Obstet Invest 1994; 38: 45-50.

8. Russell MA, Craigo MD. Cirrhosis and portal hypertension in pregnancy. Semin Perinatol 1998; 22: 156-65.

9. Ginès P, Cardenas A, Arroyo V, et al. Management of cirrhosis and ascites. N Engl J Med 2004; 350: 1646-54.

10. Sanyal AJ, Bosch J, Blei A, et al. Portal hypertension and its complications. Gastroenterology 2008; 134: 171528.

11. Schuppan D, Afdhal NH. Liver cirrhosis. Lancet 2008; 371: 838-51.

12. Runyon BA. Management of adult patients with ascites due to cirrhosis: An update. Hepatology 2009; 49: 2087-107.

13. EASL Clinical Practice Guideline. Management of ascites, spontaneous bacterial peritonitis, and hepatorenal syndrome in cirrhosis. J Hepatol 2010; 53: 397-417.

14. Errharhay S, Chaara H, Lahmine A, Oudghiri B, Ibrahimi A, Melhouf M-A, Banani A. Hypertension portale et grossesse. A propos de cinq cas et revue de la littérature. Journal Africain d'Hépato-Gastro-Entérologie, Mars 2009, volume 3, issue 1, pp 22-25.

15. Belaazri S, Lamine FZ, Siati A, Zeraidi N, Baidada A, Kharbach A. Portal Hypertension and Pregnancy: Three cases. International Annals of Medicine. 2017; 1(4). 
16. Harandou M, Ifkharen B, Madani N, Khatouf M, Kanjaa N. Hypertension portale et grossesse à propos de cinq cas. Cah Anesthesiol 2005 ; 53(2) : 93-96.

17. Maanani M, Zraidi M, Alami A, El Amrani S, Ouazzani MC. Hypertension portale et grossesse à propos de trois observations. Esperance médicale, tome $8 \mathrm{~N}^{\circ} 74: 349-351,2001$.

18. El Idrissi F, Harandou M, Mohammadi M, El-Idrissi-Lamghari K, Benabed Kn Lachkar H, Belkhadir J, Benaissa A. Hypertension portale et grossesse à propos d'une observation. Médecine du Maghreb, $\mathrm{N}^{\circ} 61,1997$.

19. Godat S, Antonio A.T, Delahvi M-A, Moradpour D, Doerig C. Hypertension portale et prise en charge de l'ascite. Rev Med Suisse $2012 ; 8: 1665-8$.

20. Tiribelli C, Rigato I. Liver cirrhosis and pregnancy. Ann Hepatol 2006; 5: 201.

21. Tan J, Surti B, Saab S. Pregnancy cirrhosis. Liver transplant 2008; 14: 1081-91.

22. Kenyon PA, Girling JC, Girling C. Liver disease in pregnancy Women's. Health Medicine 2005; 2: 26-8.

23. Crepin G, Querleu D, Bigant C, et al. Cirrhose hépatique et grossesse. Med.Chir.Dig 1977 ; $6: 127-135$.

24. Pauzner D, Wolman I, Niv D, Ber A, David MP. Endoscopic sclerotherapy in extrahepatic portal hypertension in pregnancy. Am J Obstet Gynecol 1991; 164: 152-3.

25. Pajor A, Lehoczky D. Pregnancy and extrahepatic portal hypertension. Review and report on the management. Gynecol Obstet Invest 1990; 30: 193-7.

26. Aggarwal N, Sawhney H, Vasishta K, Dhiman RK, Chawla Y. Non-cirrhotic portal hypertension in pregnancy. Int J Gynaecol Obstet 2001; 72: 1-7.

27. Lee WM. Pregnancy in patients with chronic liver disease. Gastroenterol Clin North Am 1992; 21: 889-903.

28. Bergeret S, Loffredo P, Bosson PL, Palot M, Seebacher J, Benhamou D,et al. Enquête nationale sur les alternatives à l'analgésie péridurale obstétricale. Ann F Anest Reanim $2000 ; 19: 530-9$.

29. Evron S, Glezerman M, Sadan O, Boaz M, Ezri T. Remifentanil: a novel systemic analgesic for labor pain. Anesth Analg 2005; 100: 233-8.

30. D'Alteroche L, Perarnau J-M, Perrotin F, Bacq Y. Grossesse et Hypertension portale. Gastroentérologie Clinique et Biologique 2008 ; 32 : 541-546.

31. Agence Française de Sécurité sanitaire des produits de santé. Transfusion de plasma frais congelé. Produits, indications. Recommandations, Septembre 2002. http : // agmed.sante.gouv.fr / pdf/5/rbp/tpfreco.pdf. 